\title{
THz emission from coherently controlled photocurrents in GaAs
}

\author{
D. Côtéa) and J. M. Fraser \\ Department of Physics, University of Toronto, Toronto, Ontario, Canada M5S 1A7 \\ M. DeCamp and P. H. Bucksbaum \\ Department of Physics, University of Michigan, Ann Arbor, Michigan 07974-2070 \\ H. M. van Driel \\ Department of Physics, University of Toronto, Toronto, Ontario, Canada M5S 1A7
}

(Received 21 June 1999; accepted for publication 23 October 1999)

\begin{abstract}
We report broadband terahertz radiation from ballistic photocurrents generated via quantum interference of one- and two-photon absorption in low-temperature-grown and semi-insulating GaAs at $295 \mathrm{~K}$. For 90 fs, 1550 and $775 \mathrm{~nm}$ optical pulses, we obtain phase-controllable near-single cycle $4 \mathrm{THz}$ radiation. Higher frequency THz emission should be achievable with shorter pulses. At a $250 \mathrm{kHz}$ repetition rate and average powers of $10 \mathrm{~mW}(1550 \mathrm{~nm})$ and $400 \mu \mathrm{W}(775 \mathrm{~nm})$, we measure $3 \mathrm{nW}$ of THz power, limited mainly by phase walkoff of the optical beams within the 1.5-
\end{abstract} $\mu \mathrm{m}$-thick sample and collection efficiency. (C) 1999 American Institute of Physics.

[S0003-6951(99)01751-9]

Within the last decade, there has been considerable interest in generating coherent beams at terahertz $(\mathrm{THz})$ frequencies using femtosecond laser pulses. One method to do so uses the acceleration of optically injected carriers in GaAs through, e.g., photoconductive dipole antennas, ${ }^{1,2}$ biased large apertures, ${ }^{3,4}$ or the intrinsic surface depletion field. ${ }^{5}$ With a nominally $80 \mathrm{MHz}$ Ti:sapphire laser oscillator, average $\mathrm{THz}$ powers in the nanowatt range are produced with maximum frequency $(<4 \mathrm{THz}){ }^{6}$ limited by, e.g., timevarying mobility and the onset of intervalley scattering. To achieve higher frequencies, in some cases exceeding $10 \mathrm{THz}$, optical rectification (difference frequency mixing) $)^{7-9}$ or the inverse Franz-Keldysh effect ${ }^{10}$ have been used, but the average power is considerably lower. Here, we consider an all-optical approach to high-bandwidth $\mathrm{THz}$ generation which makes use of the pulsed injection of free-carrier currents in unbiased, room-temperature bulk GaAs (band gap $E_{g}=1.4 \mathrm{eV}$ ) using coherence control (CC) concepts. In principle, the $\mathrm{THz}$ center frequency and bandwidth can be tuned by the optical pulse width and the emitted $\mathrm{THz}$ radiation can be controlled through the phases of the optical pulses.

The generation and control of electrical currents in bulk semiconductors via CC was recently proposed. ${ }^{11,12}$ For beams at $2 \omega$ and $\omega$ with $2 \hbar \omega>E_{g}>\hbar \omega$ single- and twophoton absorption processes can couple the same valenceand conduction-band states. If the beams are phase related, interference between the quantum transition amplitudes leads to a polar asymmetry in the momentum-space distribution of carriers, yielding a phase-controllable photocurrent. Within a nonlinear optics context, injection of current density occurs with $^{12}$

$$
\dot{J}_{i}^{I} \equiv\left[\frac{\partial J}{\partial t}\right]_{i}^{I}=2\left|\eta_{i j k l}\right| E_{j}^{\omega} E_{k}^{\omega} E_{l}^{2 \omega} \sin \left(2 \phi_{\omega}-\phi_{2 \omega}\right),
$$

where $E_{j, k}^{\omega}$ and $E_{l}^{2 \omega}$ are the electric-field amplitudes ${ }^{13}$ with

${ }^{a)}$ Electronic mail: dcote@physics.utoronto.ca
Cartesian indices, $i, j, k, l, \phi_{\omega}, \phi_{2 \omega}$ are their phases, and $\eta_{i j k l}$ is the total (electron plus hole) current injection tensor. Demonstration ${ }^{14}$ of $\mathrm{CC}$ currents involved measuring the integrated photocurrent of a metal-semiconductor-metal structure using low-temperature-grown (LT) GaAs or semiinsulating GaAs and femtosecond or picosecond pulse excitation. In this letter, the current generation process is directly observed through the emitted THz radiation. ${ }^{15}$

The optical source used is a $250 \mathrm{kHz}$ optical parametric amplifier which produces $90 \mathrm{fs}, 1.55 \mu \mathrm{m}(\omega)$ pulses with $40 \mathrm{~mW}$ average power. A $600-\mu \mathrm{m}$-thick beta barium borate (BBO) crystal generates up to $6 \mathrm{~mW}$ of $775 \mathrm{~nm}(1.6 \mathrm{eV})$ light $(2 \omega)$, after which the relative delay between the pulses is varied by a piezoelectrically controlled modified Michelson interferometer. The beams are copolarized and focused to a $100-\mu \mathrm{m}$-diam spot on a (100) GaAs sample (a $1.5 \mu \mathrm{m}$ epilayer of annealed LT-GaAs or $0.2-\mu \mathrm{m}$-thick semiinsulating GaAs) fastened to a microscope slide. The beams' polarization is oriented along the [100] axis of GaAs to take advantage of the largest ${ }^{12}$ tensor element, $\eta_{x x x x}$. Emitted $\mathrm{THz}$ radiation is collected from the backside with a $5 \mathrm{~cm}$ focal length, 2-cm-diam off-axis parabolic mirror after residual $\omega, 2 \omega$ radiation is removed by a low-pass filter. In one set of experiments, the collimated $\mathrm{THz}$ beam is directed to a liquid-He-cooled $\mathrm{Si}$ bolometer which measures the average $\mathrm{THz}$ power. In other experiments, the beam is first passed into a computer-controlled $\mathrm{THz}$ Michelson interferometer followed by an analyzer. ${ }^{16}$ The interferogram can be Fourier transformed to obtain the power spectrum. Lock-in detection is used via a small oscillation of the piezomounted mirror at $80 \mathrm{~Hz}$, allowing for background-free detection of the phasedependent signal.

Figure 1 shows the average $\mathrm{THz}$ power collected from the LT-GaAs sample as a function of $2 \phi_{\omega}-\phi_{2 \omega}$ for fixed optical irradiance in both beams. The data display a periodicity consistent with Eq. (1), taking into account that the far-field $\mathrm{THz}$ power is $P_{\mathrm{rad}}=\left(12 \pi \epsilon c^{3}\right)^{-1}\left[\int \dot{J} d V\right]^{2}$ where $\epsilon$ is the dielectric constant and $V$ the source volume. The peri- 


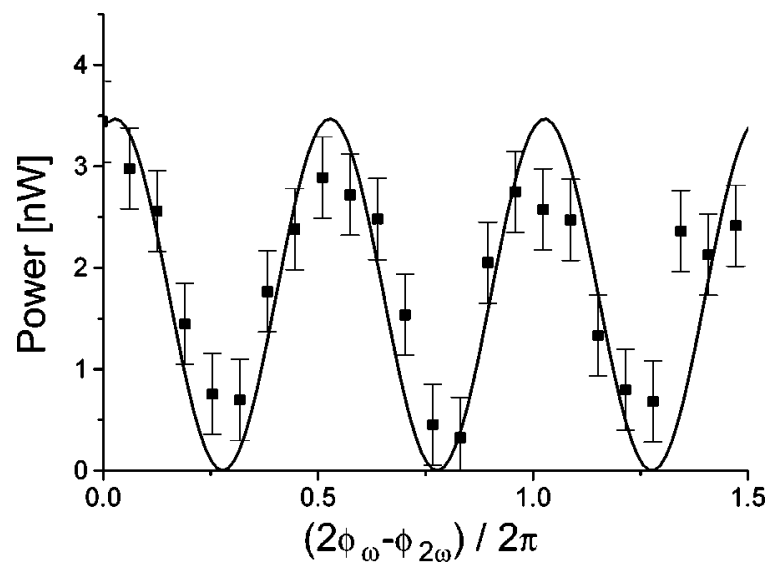

FIG. 1. Phase dependent THz power from CC currents in LT-GaAs.

odicity is independently calibrated using cascaded frequency doubling, ${ }^{17}$ wherein a second $\mathrm{BBO}$ crystal replaces the sample. Phase-dependent $\mathrm{THz}$ emission is also obtained from the semi-insulating GaAs, although the power is lower from this thinner sample.

Figure 2 shows a typical far-field interferogram of the $\mathrm{THz}$ radiation and the extracted power spectrum obtained from the LT-GaAs sample for incident pulse fluences of 400 $\mu \mathrm{J} \mathrm{cm}^{-2}(\omega)$ and $15 \mu \mathrm{J} \mathrm{cm}^{-2}(2 \omega)$. A spectral width of 3 $\mathrm{THz}$, centered at $4 \mathrm{THz}$ is indicated. Although the interferogram does not give phase information, the comparable values of bandwidth and center frequency are consistent with nearly single-cycle radiation in the THz regime. Diffraction losses could be a factor in determining the low-frequency cutoff, but we do not expect significant power below $2 \mathrm{THz}$ for reasons outlined in the next section.

A simple model for the evolution of the electron $(e)$ and hole $(h)$ carrier density $N_{e, h}$ and ballistic current density, $J_{e, h}$, reveals the underlying physics of $\mathrm{THz}$ generation. Carrier density evolution is governed by a continuity equation with carrier recombination being negligible on a $100 \mathrm{fs}$ time scale. From the momentum conservation equation with different source (injection) and dissipation (relaxation and space-charge-induced carrier deceleration) effects, one can obtain the evolution equation for the current density:

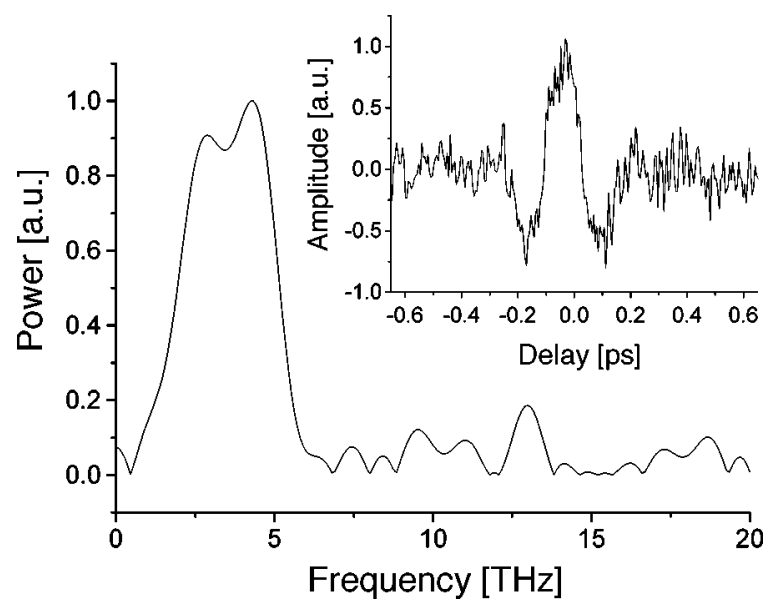

FIG. 2. THz power spectrum from currents for excitation conditions of 400 $\mu \mathrm{J} \mathrm{cm}^{-2}(\omega)$ and $15 \mu \mathrm{J} \mathrm{cm}^{-2}(2 \omega)$; the inset shows the corresponding interferogram.

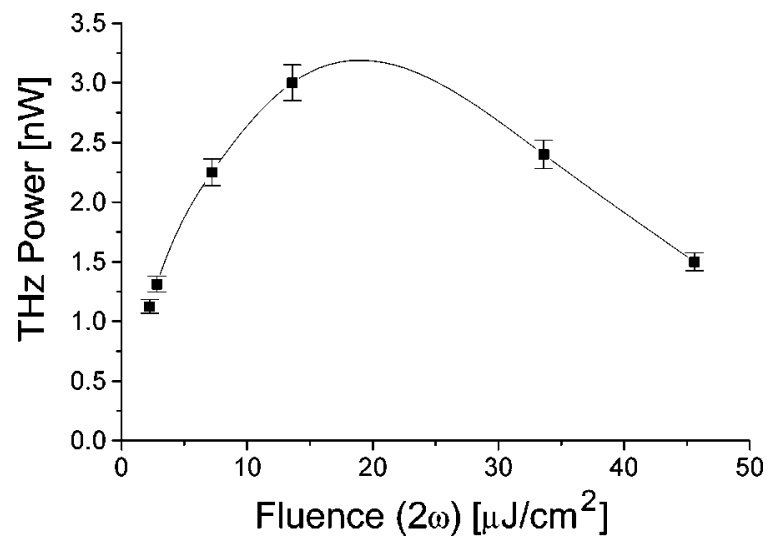

FIG. 3. Dependence of the THz average power on $775 \mathrm{~nm}$ pulse fluence for a $1550 \mathrm{~nm}$ fluence (inside sample) of about $400 \mu \mathrm{J} \mathrm{cm}^{-2}$.

$$
\frac{\partial \mathbf{J}_{e, h}}{\partial t}=\dot{\mathbf{J}}_{e, h}^{I}-\frac{\mathbf{J}_{e, h}}{\tau_{e, h}}+\frac{N_{e, h} e^{2}}{m_{e, h}^{*}} \mathbf{E}_{\mathrm{sc}},
$$

where we neglect effects that vanish under spatial integration (relevant for the total THz power radiated). Here, $m_{e, h}^{*}$ is the carrier effective mass, $\tau_{e, h}(\approx 100-200 \mathrm{fs})$ is the current relaxation time, and $E_{\mathrm{sc}}$ is the space-charge field which develops as a result of spatially inhomogeneous charge separation related to the pulses' Gaussian spatial profiles. The third term on the right-hand side, therefore, grows and opposes current flow (electron-hole separation) during and following the injection process. If we consider a simple onedimensional model in which current flows along the beams' polarization direction $(x)$, the equations for $J_{e}$ and $J_{h}$ must be solved along with Poisson equation $\epsilon \partial E_{\mathrm{sc}} / \partial x=e\left(N_{h}\right.$ $-N_{e}$ ). Although analytical solutions for $N_{e, h}$ and $J_{e, h}$ corresponding to our experimental conditions are not possible, the equations provide insight into the main features we observe.

The temporal characteristics of the current are essentially governed by the generation and relaxation terms, whereas $E_{\text {sc }}$ increasingly damps the current for high current and carrier injection. For excitation conditions below and at that corresponding to Fig. 2 with $N_{e, h}<10^{18} \mathrm{~cm}^{-3}$, the current peaks and decays with a temporal full width dominated by the optical pulse width and $\tau_{e, h}$. One, therefore, expects that $\dot{J}$, and therefore, the far-field $\mathrm{THz}$, will have a positive lobe followed by a negative lobe of approximately the same amplitude. This approximately $4 \mathrm{THz}$ single-cycle pulse, for which the bandwidth and center frequency are comparable, is consistent with the interferogram and power spectrum in Fig. 2. Note that the near-field follows $J$ and, therefore, would represent a half-cycle $\mathrm{THz}$ pulse.

To investigate the influence of space-charge effects, we investigated the scaling of $\mathrm{THz}$ emission with optical power by measuring the $\mathrm{THz}$ power as a function of $2 \omega$ fluence for an approximately constant $\omega$ fluence of $400 \mu \mathrm{J} \mathrm{cm}^{-2}$. The data, shown in Fig. 3, indicate that a maximum occurs near $15 \mu \mathrm{J} \mathrm{cm}^{-2}$ for which the average injected carrier density (dominated by the single-photon absorption) is 7 $\times 10^{17} / \mathrm{cm}^{3}$. State filling and carrier many-body effects might lead to a partial saturation of the current but cannot account for the appearance of a maximum in $\mathrm{THz}$ emission. 
For low $2 \omega$ irradiance $I_{2 \omega}$, we expect that $E_{\mathrm{sc}}$ will be negligible before current relaxation occurs so that from Eqs. (1) and (2) the THz power is proportional to $I_{2 \omega}$. At high current injection levels, the space-charge term of Eq. (2) increases faster than the injection term with $I_{2 \omega}$, leading to a maximum in $\mathrm{THz}$ emission, in agreement with the data. From another perspective, space-charge effects become important when the carrier screening time, the inverse of the plasma frequency $\left[4 \pi^{2} \epsilon m_{e}^{*} / N_{e}(t) e^{2}\right]^{1 / 2}$, drops to the pulse width. This requires $N_{e, h}=9 \times 10^{17} \mathrm{~cm}^{-3}$, in agreement with the onset of the maximum observed in Fig. 3.

We observe average $\mathrm{THz}$ powers up to $3 \mathrm{nW}$ (corresponding to a peak $\mathrm{THz}$ field of $100 \mathrm{~V} / \mathrm{cm}$ for a $100 \mu \mathrm{m}$ spot), whereas theory ${ }^{18}$ predicts a maximum value of approximately $1 \mu \mathrm{W}$ if the beams were to maintain their relative phase in the sample. Part of the discrepancy is related to the transmission of our low-pass filter $(25 \%$ at $4 \mathrm{THz})$, and the collection efficiency. However, part is also due to phase walkoff of the $\omega$ and $2 \omega$ beams [the difference in propagation constants is $\Delta k=2 \omega\left(n_{2 \omega}-n_{\omega}\right) / c=3 / \mu \mathrm{m}$, where $n$ denotes refractive index $\left.{ }^{19}\right]$ within the attenuation depth $\alpha^{-1}$ of the $2 \omega$ field $(\alpha=0.74 / \mu \mathrm{m})$, assuming that single-photon $2 \omega$ absorption determines the current decay depth. From Eq. (1), and including dephasing effects, the maximum radiated power for a sample of thickness $L$ is given by

$$
\begin{aligned}
P_{\mathrm{rad}}(\Delta k) & \propto\left|\int_{0}^{L} e^{-\alpha z} e^{i\left[\Delta k z+\left(2 \phi_{\omega}-\phi_{2 \omega}\right)_{0}\right]} d z\right|^{2} \\
& \propto \frac{1+e^{-2 \alpha L}-2 e^{-\alpha L} \cos (\Delta k L)}{\alpha^{2}+\Delta k^{2}},
\end{aligned}
$$

where $\left(2 \phi_{\omega}-\phi_{2 \omega}\right)_{0}$ is the initial phase offset. For $L=1.5 \mu \mathrm{m}, P_{\mathrm{rad}}(3 / \mu \mathrm{m}) / P_{\mathrm{rad}}(0)=0.25$, indicating that the THz power is $25 \%$ of the value achieved with $\Delta k=0$.

In summary, we have observed phase-dependent $\mathrm{THz}$ radiation from GaAs using coherently controlled currents. The phase and temporal characteristics are in agreement with the coherent control mechanism and a simple hydrodynamical model for coherent carrier transport. The output power of our radiation scheme is limited by the Manley-Rowe relations and phase mismatch, but has very few bandwidth constraints other than pump bandwidth and phonon absorption in the host material. Phase-mismatch effects could be reduced by using higher photon energies to achieve a larger $\alpha / \Delta k$ or by using an appropriately structured semiconductor to induce phase-matched beam propagation. With improvements in design, one therefore could have a pulse-width tunable and phase-controllable source of $\mathrm{THz}$ pulses.

The authors gratefully acknowledge financial support from the Natural Sciences and Engineering Research Council of Canada and Photonics Research Ontario; one of the authors (H.M.V.D.) is also appreciative of support from the Killam Program of the Canada Council. Helpful discussions with John Sipe and Peter Král and technical assistance from Tom Weinacht are much appreciated.

${ }^{1}$ P. R. Smith, D. H. Auston, and M. C. Nuss, IEEE J. Quantum Electron. 24, 255 (1988).

${ }^{2}$ M. van Exter, C. Fattinger, and D. Grischkowsky, Appl. Phys. Lett. 55, 337 (1989).

${ }^{3}$ D. You, R. Jones, P. Bucksbaum, and D. Dykaar, Opt. Lett. 18, 290 (1993).

${ }^{4}$ J. Darrow, X.-C. Zhang, and D. H. Auston, Appl. Phys. Lett. 58, 25 (1991).

${ }^{5}$ X.-C. Zhang, B. B. Hu, J. T. Darrow, and D. H. Auston, Appl. Phys. Lett. 56, 1011 (1990).

${ }^{6}$ D. Grischkowsky and N. Katzenellenbogen, Optical Society of America Proceedings on Picosecond Electronics and Optoelectronics (Optical Society of America, Washington, DC, 1991), Vol. 9, pp. 9-14.

${ }_{7}^{7}$ D. H. Auston, D. P. Cheung, J. A. Valdmanis, and D. A. Kleinman, Phys. Rev. Lett. 53, 1555 (1984).

${ }^{8}$ A. Bonvalet, M. Joffre, J. Martin, and A. Migus, Appl. Phys. Lett. 67, 2907 (1995)

${ }^{9}$ Q. Wu and X.-C. Zhang, Appl. Phys. Lett. 71, 1285 (1997).

${ }^{10}$ B. B. Hu, X.-C. Zhang, and D. H. Auston, Phys. Rev. Lett. 67, 2709 (1991).

${ }^{11}$ H. M. van Driel and A. Haché, Conference of Nonlinear Optics (Optical Society of America and IEEE, HI, 1994), Paper No. TuP21.

${ }^{12}$ R. Atanasov, A. Haché, J. Hughes, H. M. van Driel, and J. E. Sipe, Phys. Rev. Lett. 76, 1703 (1996).

${ }^{13} \mathrm{We}$ use the convention that for a monochromatic optical field $E(t)$ $=E^{\omega} \exp \left(-i \omega t+\phi_{\omega}\right)+$ c.c.

${ }^{14}$ A. Haché, Y. Kostoulas, R. Atanasov, J. Hughes, J. E. Sipe, and H. M. van Driel, Phys. Rev. Lett. 78, 306 (1997); A. Haché, J. E. Sipe, and H. M. van Driel, IEEE J. Quantum Electron. 34, 1144 (1998).

${ }^{15}$ J. Khurgin, J. Nonlinear Opt. Phys. Mater. 4, 163 (1995).

${ }^{16}$ B. I. Greene, J. F. Federici, D. R. Dykaar, R. R. Jones, and P. H. Bucksbaum, Appl. Phys. Lett. 59, 893 (1991).

${ }^{17}$ B. Y. Zel'dovich, Y. E. Kapitskii, and A. N. Chudinov, Sov. J. Quantum Electron. 20,1120 (1990) Kvant. Elektron. (Moscow) 17, 1212 (1990)].

${ }^{18}$ We use $\eta_{x x x x}=20 \mathrm{~s}^{-2} \mathrm{~m} \mathrm{C} \mathrm{V}^{-3}$ and a $2 \omega$ field attenuation coefficient $\alpha=0.74 / \mu \mathrm{m}$ to calculate $\dot{J}$ along with a $100-\mu \mathrm{m}$-diam by 1.5 - $\mu \mathrm{m}$-thick excitation volume to calculate the radiated $\mathrm{THz}$ power using the classical radiation expression in, e.g., J. D. Jackson, Classical Electrodynamics, 2nd ed. (Wiley, New York, 1975), p. 659.

${ }^{19}$ Handbook of Optical Constants of Solids, edited by E. D. Palik (Academic, Orlando, FL, 1985). 